\title{
Transformation of Highly Stable Pt Single Sites on Defect Engineered Ceria into Robust Pt Clusters for Vehicle Emission Control
}

Wei Tan, ${ }^{\text {a,b }}$ Shaohua Xie, ${ }^{\mathrm{a}}$ Yandi Cai, ${ }^{\mathrm{b}}$ Meiyu Wang,,${ }^{\mathrm{c}}$ Shuohan Yu, ${ }^{\mathrm{b}}$ Ke-Bin Low, ${ }^{\mathrm{d}}$ Yuejin Li, ${ }^{\mathrm{d}} \mathrm{Lu} \mathrm{Ma},{ }^{\mathrm{e}}$ Steven N. Ehrlich, ${ }^{\mathrm{e}}$ Fei Gao,,${ }^{\mathrm{b} *}$ Lin Dong, ${ }^{\mathrm{b}}$ Fudong Liu ${ }^{\mathrm{a}, *}$

${ }^{a}$ Department of Civil, Environmental, and Construction Engineering, Catalysis Cluster for Renewable Energy and Chemical Transformations (REACT), NanoScience Technology Center (NSTC), University of Central Florida, Orlando, FL 32816, United States

${ }^{b}$ Key Laboratory of Mesoscopic Chemistry of MOE, School of Chemistry and Chemical Engineering, Jiangsu Key Laboratory of Vehicle Emissions Control, School of Environment, Center of Modern Analysis, Nanjing University, Nanjing 210023, P. R. China

${ }^{c}$ College of Engineering and Applied Sciences, Nanjing University, Nanjing, 210023, P. R. China

${ }^{d}$ BASF Corporation, Iselin, NJ 08830, United States

${ }^{e}$ National Synchrotron Light Source II (NSLS-II), Brookhaven National Laboratory, Upton, NY 11973, United States

\section{*Corresponding Authors}

gaofei@nju.edu.cn (F. Gao); fudong.liu@ucf.edu (F. Liu) 


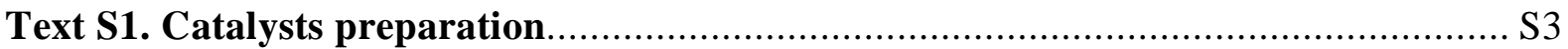

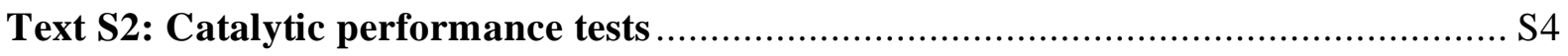

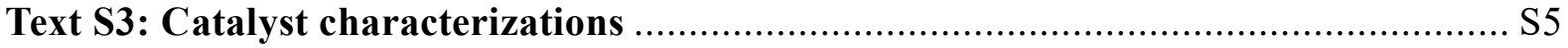

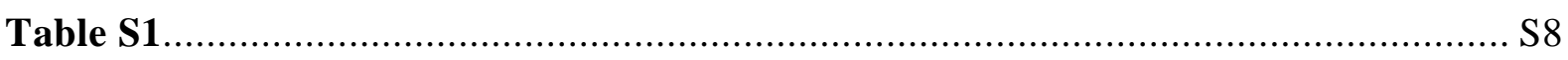

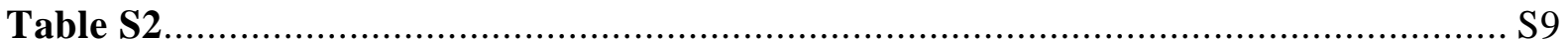

Table S3

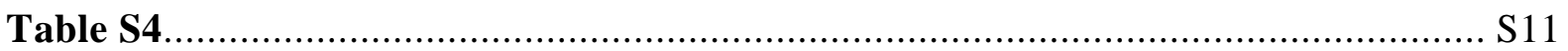

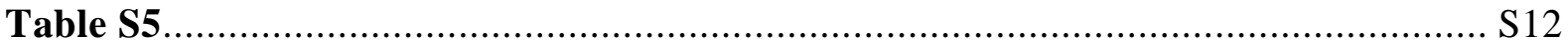

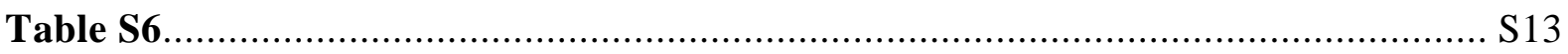

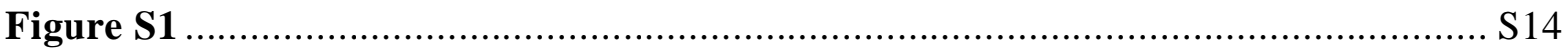

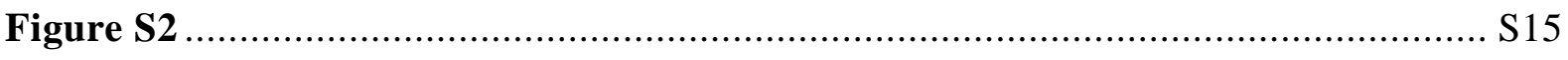

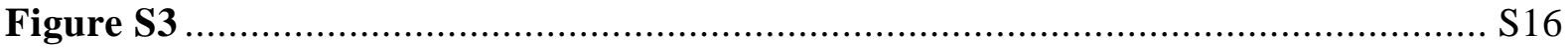

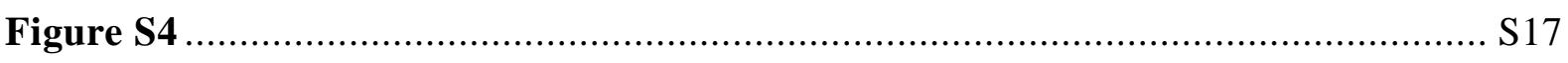

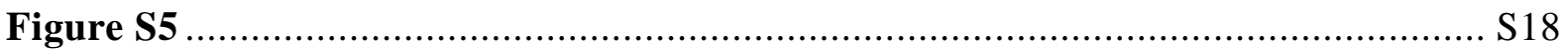

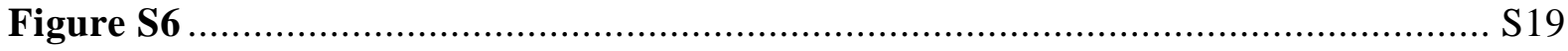

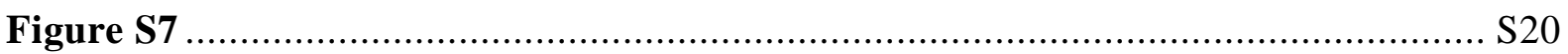

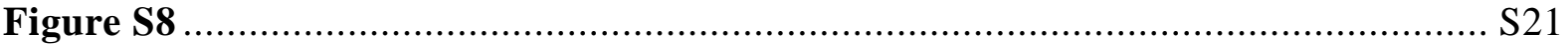

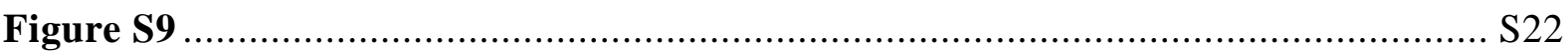

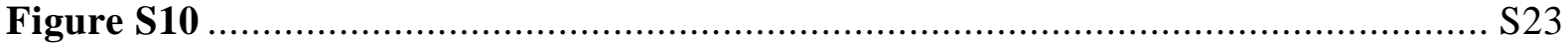

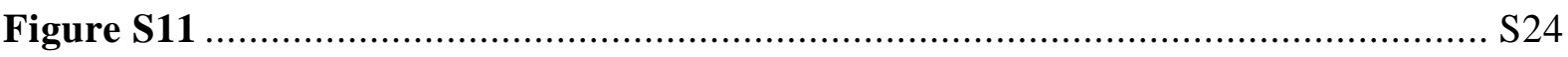

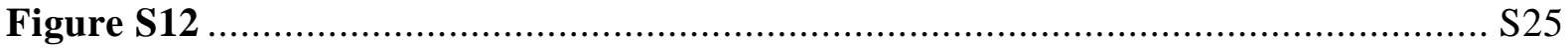

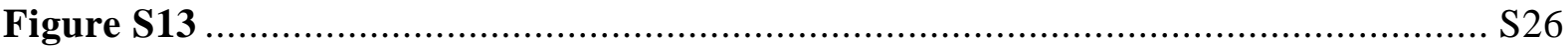

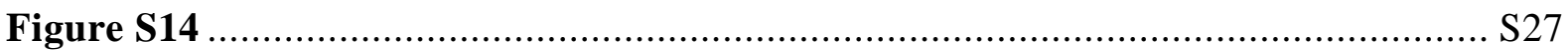

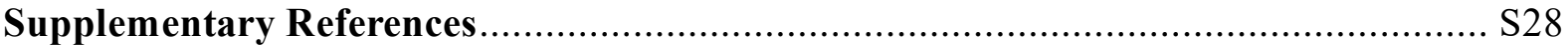




\section{Supporting Information}

\section{Text S1: Catalysts preparation}

For the preparation of pristine $\mathrm{CeO}_{2}$, a certain amount of $\mathrm{Ce}\left(\mathrm{NO}_{3}\right)_{3} \cdot 6 \mathrm{H}_{2} \mathrm{O}$ was dissolved in deionized water. Excess $\mathrm{NH}_{3} \cdot \mathrm{H}_{2} \mathrm{O}$ was added to the solution under vigorous stirring until the $\mathrm{pH}$ of the solution reached 10 . Then, the resulted mixture was aged overnight, filtered, and washed with deionized water for several times. The obtained precipitate was dried at $100{ }^{\circ} \mathrm{C}$ for $24 \mathrm{~h}$ and subsequently calcined at $550{ }^{\circ} \mathrm{C}$ for $2 \mathrm{~h}$ in air with a ramping rate of $5 \mathrm{~K} / \mathrm{min}$. To prepare $\mathrm{Ce}_{0.9} \mathrm{Zr}_{0.1} \mathrm{O}_{2}, \mathrm{Ce}\left(\mathrm{NO}_{3}\right)_{3} \cdot 6 \mathrm{H}_{2} \mathrm{O}$ and $\mathrm{ZrO}\left(\mathrm{NO}_{3}\right)_{2}$ with the molar ratio of $9: 1$ were used as precursors, with other procedures kept the same for the synthesis of pristine $\mathrm{CeO}_{2}$. The obtained $\mathrm{Zr}$ doped $\mathrm{CeO}_{2}$ was denoted as $\mathrm{CZO}$. 1 wt.\% Pt was loaded on $\mathrm{CeO}_{2}$ and $\mathrm{Ce}_{0 .} \mathrm{Zr}_{0.1} \mathrm{O}_{2}$ using incipient wetness impregnation (IWI) method, where tetraamineplatinum(II) nitrate $\left(\mathrm{Pt}\left(\mathrm{NH}_{3}\right)_{4}\left(\mathrm{NO}_{3}\right)_{2}\right.$, TAPN) obtained from Alfa Aesar was used as Pt precursor. After the impregnation of TAPN solution, the obtained powders were dried at $120{ }^{\circ} \mathrm{C}$ for $20 \mathrm{~min}$ and then calcined at $550{ }^{\circ} \mathrm{C}$ for $2 \mathrm{~h}$ in air. The obtained samples were denoted as $\mathrm{Pt} / \mathrm{CeO}_{2}$ and $\mathrm{Pt} / \mathrm{CZO}$. Pt catalysts supported on $\mathrm{Ce}_{x} \mathrm{Zr}_{1-x} \mathrm{O}_{2}$ with different $\mathrm{Zr}$ molar ratios $(x=0.95,0.85,0.6$ and 0.3), $\mathrm{CeO}_{2}$ nanorod (CeNR), commercial high surface area (HSA) $\mathrm{CeO}_{2}\left(c a .120 \mathrm{~m}^{2} / \mathrm{g}\right.$ ) and commercial $\mathrm{CeZrO}_{x}\left(10\right.$ wt.\% $\left.\mathrm{ZrO}_{2}\right)$ were also prepared as references. $\mathrm{Ce}_{x} \mathrm{Zr}_{1-x} \mathrm{O}_{2}$ was prepared by the co-precipitation method as mentioned above. CeNR was synthesized by a typical hydrothermal method according to the literature [1]. The commercial $\mathrm{CeO}_{2}$-based supports in this work were provided by BASF Corporation. 


\section{Supporting Information}

\section{Text S2: Catalytic performance tests}

The $\mathrm{CO}$ oxidation and $\mathrm{C}_{3} \mathrm{H}_{6}$ oxidation activities were evaluated in a fixed-bed quartz tube reactor consisted of a tube furnace and a straight quartz tube. For each test, $25 \mathrm{mg}$ sample (40$60 \mathrm{mesh}$ ) was pre-mixed with $250 \mathrm{mg} \mathrm{SiC}$, and the mixture was loaded in the middle of the quartz tube. An online thermocouple was placed adjacent to the sample outside the quartz tube. For $\mathrm{CO}$ oxidation activity test, the feeding gas consisted of $1 \% \mathrm{CO}$ and $1 \% \mathrm{O}_{2}$ (Ar as balance), and the reaction was performed under steady state conditions. For $\mathrm{C}_{3} \mathrm{H}_{6}$ oxidation test, the feeding gas consisted of $2000 \mathrm{ppm}_{3} \mathrm{H}_{6}$ and $2 \% \mathrm{O}_{2}$, and the reaction was performed under transient conditions with a temperature ramping rate of $2 \mathrm{~K} / \mathrm{min}$. For all tests, the total flow rate was $83.3 \mathrm{~mL} / \mathrm{min}$ with a weight hourly space velocity (WHSV) of $200,000 \mathrm{~mL} \cdot \mathrm{g}_{\text {cat. }}{ }^{-1} \cdot \mathrm{h}^{-1}$. The outlet gas was detected by an online mass spectrometer (Hiden Analytical, HPR20 R\&D). The mass to charge $(\mathrm{m} / \mathrm{z})$ ratios used for the detection of $\mathrm{CO}, \mathrm{CO}_{2}$ and $\mathrm{C}_{3} \mathrm{H}_{6}$ were 28,44 and 41 , respectively. 


\section{Supporting Information}

\section{Text S3: Catalyst characterizations}

A Philips X'pert Pro diffractometer with Ni filtered $\mathrm{Cu} K \alpha 1$ radiation $(0.15408 \mathrm{~nm})$ was used to collect the X-ray powder diffraction (XRD) patterns. The X-ray tube was operated at $40 \mathrm{kV}$ and $40 \mathrm{~mA}$. The XRD patterns were collected over a $2 \theta$ range of $20-80^{\circ}$. The scan step was $0.02^{\circ}$ with a scan speed of $10 \%$ min.

Raman spectra were collected on a Spex $1877 \mathrm{D}$ triplemate spectrograph with $2 \mathrm{~cm}^{-1}$ resolution at room temperature. The wavenumber of the laser was $532 \mathrm{~nm}$ with a power output of $5 \mathrm{~mW}$.

In situ diffuse reflectance infrared Fourier transform spectroscopy (in situ DRIFTS) was collected on a Nicolet iS50 FTIR spectrometer equipped with an MCT detector cooled by liquid $\mathrm{N}_{2}$. In each test, $c a .50 \mathrm{mg}$ sample was loaded in a sample cell, pressed and mounted. Then, the samples were pretreated by air at $300{ }^{\circ} \mathrm{C}$ or $10 \% \mathrm{H}_{2} / \mathrm{Ar}$ at $400{ }^{\circ} \mathrm{C}$ for $1 \mathrm{~h}$. Afterwards, the samples were cooled to room temperature. The background spectra of the catalysts were collected at specific temperatures during the cooling process. Methanol adsorption experiments were performed at $25{ }^{\circ} \mathrm{C}$. The feeding gas was set as $15 \% \mathrm{CH}_{3} \mathrm{OH}$ in Ar balance $(83.33$ $\mathrm{mL} / \mathrm{min}$ ). For $\mathrm{CO}$ adsorption/oxidation experiments, the feed gas was set as $1 \% \mathrm{CO}$ when needed, $1 \% \mathrm{O}_{2}$ when needed, $\mathrm{Ar}$ as balance $(83.33 \mathrm{~mL} / \mathrm{min})$. The spectra were collected from 400 to $4000 \mathrm{~cm}^{-1}$ with a resolution of $4 \mathrm{~cm}^{-1}$ for 100 scans.

Atomic-resolution high angle annular dark-field scanning transmission electron microscopic (HAADF-STEM) images were collected on an FEI Titan Cubed G2 60-300 aberrationcorrected S/TEM instrument at $300 \mathrm{kV}$ accelerating voltage. The observations were performed 


\section{Supporting Information}

in the high angle annular dark-field (HAADF) mode, which allowed Z-contrast imaging. The probe convergence angle on the Titan electron microscope was $22 \mathrm{mrad}$, and the angular range of the HAADF detector was from 79.5 to $200 \mathrm{mrad}$. To prepare the TEM samples, an appropriate amount of the sample powder was dispersed in ethanol and then dropped on a 3 mm TEM Mo grid. The images of energy dispersive spectroscopy (EDS) elemental mapping in the STEM mode were obtained from the Titan electron microscope using SuperX system.

The composition of the prepared catalysts was determined by an inductively coupled plasmaoptical emission spectrometer (ICP-OES, Avio 500, PerkinElmer).

X-ray absorption spectroscopy (XAS) for Pt $\mathrm{L}_{3}$-edge was measured in fluorescence mode at 7-BM QAS beamline of the National Synchrotron Light Source II (NSLS-II) at Brookhaven National Laboratory. The beamline could provide X-ray with energies ranging from 4.7 to 31 $\mathrm{keV}$. The monochromator equipped with a $\mathrm{Si}(111)$ channel-cut crystal runs at continuous scan mode. A typical scan time was $40 \mathrm{~s}$ and each sample was scanned 20 times. The XAS data including X-ray absorption near edge structure (XANES) and extended X-ray absorption fine structure (EXAFS) were analyzed using Demeter software package. Pt foil was applied for energy calibration and drift correction of the monochromator.

The specific surface areas of prepared samples were measured by $\mathrm{N}_{2}$-physisorption at -196 ${ }^{\circ} \mathrm{C}$ on a Micromeritics ASAP-2020 analyzer, using Brunaeur, Emmett and Teller (BET) method. Before each test, the samples were degassed at $300{ }^{\circ} \mathrm{C}$ for $3 \mathrm{~h}$.

$\mathrm{H}_{2}$-temperature programmed reduction $\left(\mathrm{H}_{2}-\mathrm{TPR}\right)$ of prepared samples was conducted on a Quantachrome Autosorb-iQ instrument. In each test, $30 \mathrm{mg}$ of the sample was first treated with 
air at $400{ }^{\circ} \mathrm{C}$ for $1 \mathrm{~h}$. Then, the sample was cooled down to room temperature in air. After switching the purging gas to $\mathrm{H}_{2}\left(10 \% \mathrm{H}_{2} / \mathrm{Ar}, 30 \mathrm{~mL} / \mathrm{min}\right)$, the temperature was linearly raised from 30 to $900{ }^{\circ} \mathrm{C}$ with a ramping rate of $10 \mathrm{~K} / \mathrm{min}$. The signal of $\mathrm{H}_{2}$ consumption was recorded by a thermal conductivity detector (TCD).

X-ray photoelectron spectra (XPS) of prepared samples were collected on a PHI 5000 VersaProbe system, with monochromatic $\mathrm{K} \alpha$ radiation $(1486.6 \mathrm{eV})$ operated at an accelerating power of $15 \mathrm{~kW}$. All binding energies of prepared samples were calibrated with the adventitious C1s peak at $284.6 \mathrm{eV}$.

The dynamic oxygen storage capacity (OSC) was measured by a multi-pulse experiment under alternating pulses of $2 \% \mathrm{CO} / 4 \% \mathrm{O}_{2}$ with a total flow rate of $100 \mathrm{~mL} / \mathrm{min}$. Before testing, all samples were activated by $10 \% \mathrm{H}_{2} / \mathrm{Ar}$ at $400{ }^{\circ} \mathrm{C}$ for $1 \mathrm{~h}$. For each temperature point, the testing duration was $30 \mathrm{~min}$. A single cycle lasted $1 \mathrm{~min}$, with $30 \mathrm{~s} \mathrm{CO} / 30 \mathrm{~s} \mathrm{O} 2$ cycling switch controlled by MFC systems. The OSC values were calculated from the average $\mathrm{CO}_{2}$ formation detected by an online mass spectrometer (Hiden Analytical, HPR20 R\&D). 
Table S1. Crystalline size, lattice constant and heigh of diffraction peak based on XRD patterns of $\mathrm{CeO}_{2}$ and $\mathrm{CZO}$.

\begin{tabular}{cccc}
\hline Samples & $\begin{array}{c}\text { Crystalline size } \\
(\mathbf{n m})\end{array}$ & $\begin{array}{c}\text { Lattice constant } \\
(\mathbf{n m})\end{array}$ & $\begin{array}{c}\text { Height of diffraction peak } \\
\left(\mathbf{C e O}_{2}[\mathbf{1 1 1}]\right)(\mathbf{a . u .})\end{array}$ \\
\hline $\mathbf{C e O}_{2}$ & 13.2 & 0.53975 & 225 \\
$\mathbf{C Z O}$ & 12.2 & 0.53858 & 178 \\
\hline
\end{tabular}


Table S2. Data summary on the parameter of in situ DRIFTS of CO adsorption

\begin{tabular}{|c|c|c|c|c|c|c|}
\hline Samples & $\begin{array}{l}\text { Position } \\
\left(\mathrm{cm}^{-1}\right)\end{array}$ & $\begin{array}{c}\text { FWHM } \\
\left(\mathrm{cm}^{-1}\right)\end{array}$ & $\begin{array}{c}\text { Peak } \\
\text { Height } \\
\text { (a.u.) }\end{array}$ & $\begin{array}{c}\text { Ratio } \\
\text { decrease } \\
(\%)^{a}\end{array}$ & $\begin{array}{l}\text { Peak } \\
\text { Area } \\
\text { (a.u.) }\end{array}$ & $\begin{array}{c}\text { Ratio } \\
\text { decrease } \\
(\%)^{b}\end{array}$ \\
\hline $\mathrm{Pt} / \mathrm{CeO}_{2}$ & 2094 & 26.6 & 0.130 & / & 3.97 & l \\
\hline $\mathrm{Pt} / \mathrm{CZO}$ & 2097 & 27.4 & 0.234 & l & 7.77 & l \\
\hline $\mathrm{Pt} / \mathrm{CeO}_{2}-800 \mathrm{~A}$ & 2089 & 19.1 & 0.06 & 54.6 & 1.37 & 65.5 \\
\hline Pt/CZO-800A & 2090 & 20.3 & 0.138 & 41.0 & 3.40 & 56.2 \\
\hline
\end{tabular}

${ }^{a}$ The ratio decrease of peak height before and after aging treatment.

${ }^{\mathrm{b}}$ The ratio decrease of peak area before and after aging treatment. 
Table S3. BET surface area, surface element concentration and chemical states of prepared catalysts.

\begin{tabular}{|c|c|c|c|c|c|c|c|}
\hline \multirow{2}{*}{ Samples } & \multirow{2}{*}{$\begin{array}{l}\text { BET surface } \\
\operatorname{area} /\left(\mathrm{m}^{2} / \mathrm{g}\right)^{\mathrm{a}}\end{array}$} & \multicolumn{4}{|c|}{ Surface atomic concentration $(\%)$} & \multirow{2}{*}{$\begin{array}{l}\text { Pt loading } \\
\left(w t^{0}\right)^{b}\end{array}$} & \multirow{2}{*}{$\begin{array}{c}\text { Mole } \\
\text { fraction of } \\
\operatorname{Zr}(\%)^{b}\end{array}$} \\
\hline & & $\mathbf{O}$ & $\mathbf{Z r}$ & $\mathrm{Ce}$ & $\mathbf{P t}$ & & \\
\hline $\mathrm{CeO}_{2}$ & 44 & 71.5 & - & 28.5 & - & - & - \\
\hline $\mathrm{CZO}$ & 59 & 72.0 & 3.5 & 24.0 & - & - & - \\
\hline $\mathrm{Pt} / \mathrm{CeO}_{2}$ & 42 & 71.3 & - & 27.8 & 0.88 & - & - \\
\hline $\mathrm{Pt} / \mathrm{CZO}$ & 58 & 70.3 & 4.0 & 24.7 & 1.01 & - & - \\
\hline $\mathrm{Pt} / \mathrm{CeO}_{2}-800 \mathrm{~A}$ & 24 & 71.6 & - & 27.2 & 1.15 & 0.97 & - \\
\hline $\mathrm{Pt} / \mathrm{CZO}-800 \mathrm{~A}$ & 39 & 70.9 & 3.0 & 24.9 & 1.12 & 0.98 & 9.6 \\
\hline $\mathrm{Pt} / \mathrm{CeO}_{2}-\mathrm{a}$ & I & 73.4 & - & 26.1 & 0.53 & - & - \\
\hline $\mathrm{Pt} / \mathrm{CZO}-\mathrm{a}$ & I & 73.7 & 4.2 & 21.5 & 0.58 & - & - \\
\hline $\mathrm{Pt} / \mathrm{CeO}_{2}-800 \mathrm{~A}-\mathrm{a}$ & I & 72.2 & - & 27.1 & 0.69 & - & - \\
\hline Pt/CZO-800A-a & I & 75.0 & 3.9 & 20.4 & 0.73 & - & - \\
\hline
\end{tabular}

a The specific surface areas of as-prepared samples were measured by $\mathrm{N}_{2}$ physisorption at -196 ${ }^{\circ} \mathrm{C}$.

${ }^{b}$ The Pt loading in $\mathrm{Pt} / \mathrm{CeO}_{2}-800 \mathrm{~A}$ and $\mathrm{Pt} / \mathrm{CZO}-800 \mathrm{~A}$, and the mole fraction of $\mathrm{Zr}$ in $\mathrm{Pt} / \mathrm{CZO}-$ $800 \mathrm{~A}$ were determined by ICP-OES. 
Table S4. State of $\mathrm{Pt}$ species, $\mathrm{T}_{50}, \mathrm{~T}_{90}$ and reaction rate for $\mathrm{CO}$ oxidation on $\mathrm{Pt} / \mathrm{CZO}-\mathrm{a}$, $\mathrm{Pt} / \mathrm{CZO}-800 \mathrm{~A}-\mathrm{a}$ and recently reported $\mathrm{Pt}-\mathrm{CeO}_{2}$ based catalysts.

\begin{tabular}{|c|c|c|c|c|c|c|}
\hline Catalysts ${ }^{a}$ & State of Pt & $\begin{array}{l}\mathrm{T}_{50} \\
\left({ }^{\circ} \mathrm{C}\right)\end{array}$ & $\begin{array}{l}\mathrm{T}_{90} \\
\left({ }^{\circ} \mathrm{C}\right)\end{array}$ & $\begin{array}{l}\text { Reaction rate at } 80^{\circ} \mathrm{C} \\
\left(\mathrm{mmol} \cdot \mathrm{g}_{\mathrm{Pt}}{ }^{-1} \cdot \mathrm{s}^{-1}\right)^{\mathrm{b}}\end{array}$ & $\begin{array}{c}\text { WHSV } \\
\left(\mathrm{mL} \cdot \mathrm{g}_{\mathrm{cat}}{ }^{-1} \cdot \mathrm{h}^{-1}\right)\end{array}$ & Ref \\
\hline $\mathrm{Pt} / \mathrm{CZO}-\mathrm{a}$ & Clusters & 86 & 123 & $\begin{array}{c}1.02 \\
\text { (Steady state) }\end{array}$ & 200,000 & / \\
\hline $\mathrm{Pt} / \mathrm{CZO}-800 \mathrm{~A}-\mathrm{a}$ & Clusters & 92 & 117 & $\begin{array}{c}0.60 \\
\text { (Steady state) }\end{array}$ & 200,000 & l \\
\hline $\begin{array}{l}\mathrm{Pt} / \mathrm{CeO}_{2} \text { nanocubes } \\
\left(0.3 \mathrm{wt} \% \mathrm{Pt} / \mathrm{CeO}_{2}\right)\end{array}$ & clusters & 176 & 179 & $\begin{array}{c}0.10 \\
\left(10^{\circ} \mathrm{C} / \mathrm{min}\right)\end{array}$ & 120,000 & {$[2]$} \\
\hline $\begin{array}{c}\mathrm{Pt} / \mathrm{CeO}_{2} \mathrm{~S}_{-} \\
\left(1 \mathrm{wt} \% \mathrm{Pt} / \mathrm{CeO}_{2}\right)\end{array}$ & Single atoms & 135 & 149 & $\begin{array}{c}0.05 \\
\left(2{ }^{\circ} \mathrm{C} / \mathrm{min}\right)\end{array}$ & 200,000 & [3] \\
\hline $\begin{array}{c}\mathrm{Ce}_{0.93} \mathrm{Zn}_{0.05} \mathrm{Pt}_{0.02} \mathrm{O}_{2-\delta} \\
\left(2.3 \mathrm{wt} \% \mathrm{Pt} / \mathrm{Ce}_{0.93} \mathrm{Zn}_{0.05} \mathrm{O}_{x}\right)\end{array}$ & Particles & 134 & 150 & $\begin{array}{c}0.03 \\
\text { (Steady state) }\end{array}$ & 120,000 & {$[4]$} \\
\hline $\begin{array}{c}\text { Activated AT } \\
\left(1 \mathrm{wt} \% \mathrm{Pt} / \mathrm{CeO}_{2}\right)\end{array}$ & Clusters & 56 & 64 & $\begin{array}{c}/ \\
\left(2{ }^{\circ} \mathrm{C} / \mathrm{min}\right)\end{array}$ & 232,500 & {$[5]$} \\
\hline $\begin{array}{c}\mathrm{Pt} / \mathrm{CeBTC}-1 \\
\left(3.12 \mathrm{wt} \% \mathrm{Pt} / \mathrm{CeO}_{2}\right)\end{array}$ & Clusters & 43 & 64 & $\begin{array}{c}0.07 \\
\left(5^{\circ} \mathrm{C} / \mathrm{min}\right)\end{array}$ & 18,000 & {$[6]$} \\
\hline $\begin{array}{c}\mathrm{N}-\mathrm{Pt} / \mathrm{CeO}_{2} \\
\left(1.33 \mathrm{wt} \% \mathrm{Pt} / \mathrm{CeO}_{2}\right)\end{array}$ & Clusters & 60 & 92 & $\begin{array}{c}0.46 \\
\left(2{ }^{\circ} \mathrm{C} / \mathrm{min}\right)\end{array}$ & 60,000 & {$[7]$} \\
\hline $\begin{array}{c}\text { Pt-SA-Ce-MOF } \\
\left(0.12 \mathrm{wt} \% \mathrm{Pt} / \mathrm{CeO}_{2}\right)\end{array}$ & Single atoms & 143 & 150 & $\begin{array}{c}0.10 \\
\left(10^{\circ} \mathrm{C} / \mathrm{min}\right)\end{array}$ & 120,000 & [8] \\
\hline $\begin{array}{c}1 \mathrm{PtCT} \\
\left(1 \mathrm{wt} \% \mathrm{Pt} / \mathrm{CeO}_{2}-\mathrm{TiO}_{2}\right)\end{array}$ & Clusters & 135 & 152 & $\begin{array}{c}0.03 \\
\left(3^{\circ} \mathrm{C} / \mathrm{min}\right)\end{array}$ & 60,000 & {$[9]$} \\
\hline $\begin{array}{c}\text { Meso } \mathrm{Pt} / \mathrm{Ce}_{0.8} \mathrm{Zr}_{0.2} \mathrm{O}_{2} \\
\left(0.4 \mathrm{wt} \% \mathrm{Pt} / \mathrm{Ce}_{0.8} \mathrm{Zr}_{0.2} \mathrm{O}_{2}\right)\end{array}$ & Particles & 126 & 134 & $\begin{array}{c}0.09 \\
\left(1^{\circ} \mathrm{C} / \mathrm{min}\right)\end{array}$ & 60,000 & {$[10]$} \\
\hline
\end{tabular}

${ }^{a}$ The specific compositions of the catalysts can be found in the parentheses.

${ }^{\mathrm{b}}$ The reactor heating modes (steady state $v s$. linear ramping) in the catalytic activity test were marked in parentheses. 
Table S5. $\mathrm{H}_{2}$ consumption of the prepared catalysts calculated based on the $\mathrm{H}_{2}-\mathrm{TPR}$

experiment

\begin{tabular}{lcccc}
\hline \multirow{2}{*}{ Samples } & \multicolumn{4}{c}{ H$_{2}$-consumption amount $\left(\mathbf{m m o l} / \mathbf{g}_{\text {cat. }}\right)$} \\
\cline { 2 - 5 } & $\boldsymbol{\alpha}$ & $\boldsymbol{\beta}$ & $\boldsymbol{\gamma}$ & $\boldsymbol{\delta}$ \\
\hline $\mathrm{CeO}_{2}$ & 0.20 & 0.30 & 1.12 & - \\
$\mathrm{CZO}$ & 0.26 & 0.31 & 1.18 & - \\
$\mathrm{Pt} / \mathrm{CeO}_{2}$ & - & 0.11 & 0.86 & 0.22 \\
$\mathrm{Pt} / \mathrm{CZO}$ & - & 0.25 & 1.06 & 0.41 \\
$\mathrm{Pt} / \mathrm{CeO} 2-800 \mathrm{~A}$ & - & 0.05 & 1.08 & 0.21 \\
$\mathrm{Pt} / \mathrm{CZO}-800 \mathrm{~A}$ & - & 0.35 & 1.01 & 0.40 \\
\hline
\end{tabular}


Table S6. EXAFS fitting results for aged and activated Pt-based catalysts using $\mathrm{Pt}$ foil, $\mathrm{PtO}_{2}$ as references $\left(k\right.$ range $=3.0-12.0 \AA^{-1}, \mathrm{Rbkg}=1.0$ for Fourier transform $)$.

\begin{tabular}{|c|c|c|c|c|c|c|}
\hline Sample & Shell & $\mathrm{CN}^{\mathrm{a}}$ & $\mathbf{R}(\AA)^{\mathbf{b}}$ & $\Delta E_{0}(e V)^{c}$ & $\sigma^{2}\left(10^{-3} \AA^{2}\right)^{d}$ & R-factor ${ }^{\mathrm{e}}$ \\
\hline \multirow[t]{2}{*}{$\mathrm{Pt}$} & $\mathrm{Pt}-\mathrm{Pt}$ & $9.5 \pm 0.4$ & $2.76 \pm 0.01$ & $8.1 \pm 0.3$ & $4.38 \pm 0.25$ & 0.001 \\
\hline & $\mathrm{Pt}-\mathrm{O}$ & $5.1 \pm 0.4$ & $2.01 \pm 0.01$ & $8.9 \pm 1.0$ & $2.69 \pm 0.92$ & \multirow{3}{*}{0.007} \\
\hline \multirow[t]{2}{*}{$\mathrm{PtO}_{2}$} & $\mathrm{Pt}-\mathrm{O}-\mathrm{Pt}$ & $5.1 \pm 0.4$ & $3.08 \pm 0.01$ & $5.2 \pm 3.0$ & $3.13 \pm 0.72$ & \\
\hline & Pt-O & $10.2 \pm 0.8$ & $3.73 \pm 0.03$ & $14.7 \pm 1.8$ & $6.12 \pm 3.12$ & \\
\hline \multirow{2}{*}{$\mathrm{Pt} / \mathrm{CeO}_{2}-800 \mathrm{~A}$} & $\mathrm{Pt}-\mathrm{O}$ & $3.4 \pm 0.4$ & $2.00 \pm 0.01$ & $8.4 \pm 1.1$ & $0.24 \pm 1.17$ & \multirow{2}{*}{0.028} \\
\hline & $\mathrm{Pt}-\mathrm{O}-\mathrm{Ce}$ & $2.2 \pm 1.6$ & $3.10 \pm 0.05$ & $-3.0 \pm 5.7$ & $7.78 \pm 5.36$ & \\
\hline \multirow{2}{*}{$\mathrm{Pt} / \mathrm{CZO}-800 \mathrm{~A}$} & $\mathrm{Pt}-\mathrm{O}$ & $3.6 \pm 0.4$ & $2.00 \pm 0.01$ & $8.5 \pm 1.4$ & $1.35 \pm 1.27$ & \multirow{2}{*}{0.013} \\
\hline & $\mathrm{Pt}-\mathrm{O}-\mathrm{Ce}$ & $3.0 \pm 1.6$ & $3.07 \pm 0.04$ & $-4.5 \pm 4.6$ & $8.69 \pm 4.21$ & \\
\hline \multirow{3}{*}{$\mathrm{Pt} / \mathrm{CeO}_{2}-800 \mathrm{~A}-\mathrm{a}$} & Pt-O & $2.6 \pm 0.4$ & $2.01 \pm 0.01$ & $8.4 \pm 1.1$ & $3.34 \pm 2.20$ & \multirow{3}{*}{0.036} \\
\hline & $\mathrm{Pt}-\mathrm{Pt}$ & $1.2 \pm 0.6$ & $2.64 \pm 0.04$ & $8.1 \pm 0.3$ & $5.15 \pm 8.11$ & \\
\hline & $\mathrm{Pt}-\mathrm{O}-\mathrm{Ce}$ & $0.7 \pm 1.2$ & $3.11 \pm 0.06$ & $-4.5 \pm 4.6$ & $6.15 \pm 11.35$ & \\
\hline \multirow{3}{*}{$\mathrm{Pt} / \mathrm{CZO}-800 \mathrm{~A}-\mathrm{a}$} & Pt-O & $3.0 \pm 0.2$ & $2.01 \pm 0.01$ & $8.5 \pm 1.4$ & $3.26 \pm 1.95$ & \multirow{3}{*}{0.034} \\
\hline & $\mathrm{Pt}-\mathrm{Pt}$ & $0.8 \pm 1.0$ & $2.64 \pm 0.03$ & $8.1 \pm 0.3$ & $5.15 \pm 8.11$ & \\
\hline & $\mathrm{Pt}-\mathrm{O}-\mathrm{Ce}$ & $1.1 \pm 0.7$ & $3.12 \pm 0.04$ & $-4.5 \pm 4.6$ & $8.69 \pm 4.21$ & \\
\hline
\end{tabular}

a Coordination number; ${ }^{\mathrm{b}}$ Bond length; ${ }^{\mathrm{c}}$ Energy shift; ${ }^{\mathrm{d}}$ Debye-Waller factor; ${ }^{\mathrm{e}}$ Goodness-of-fit index. 

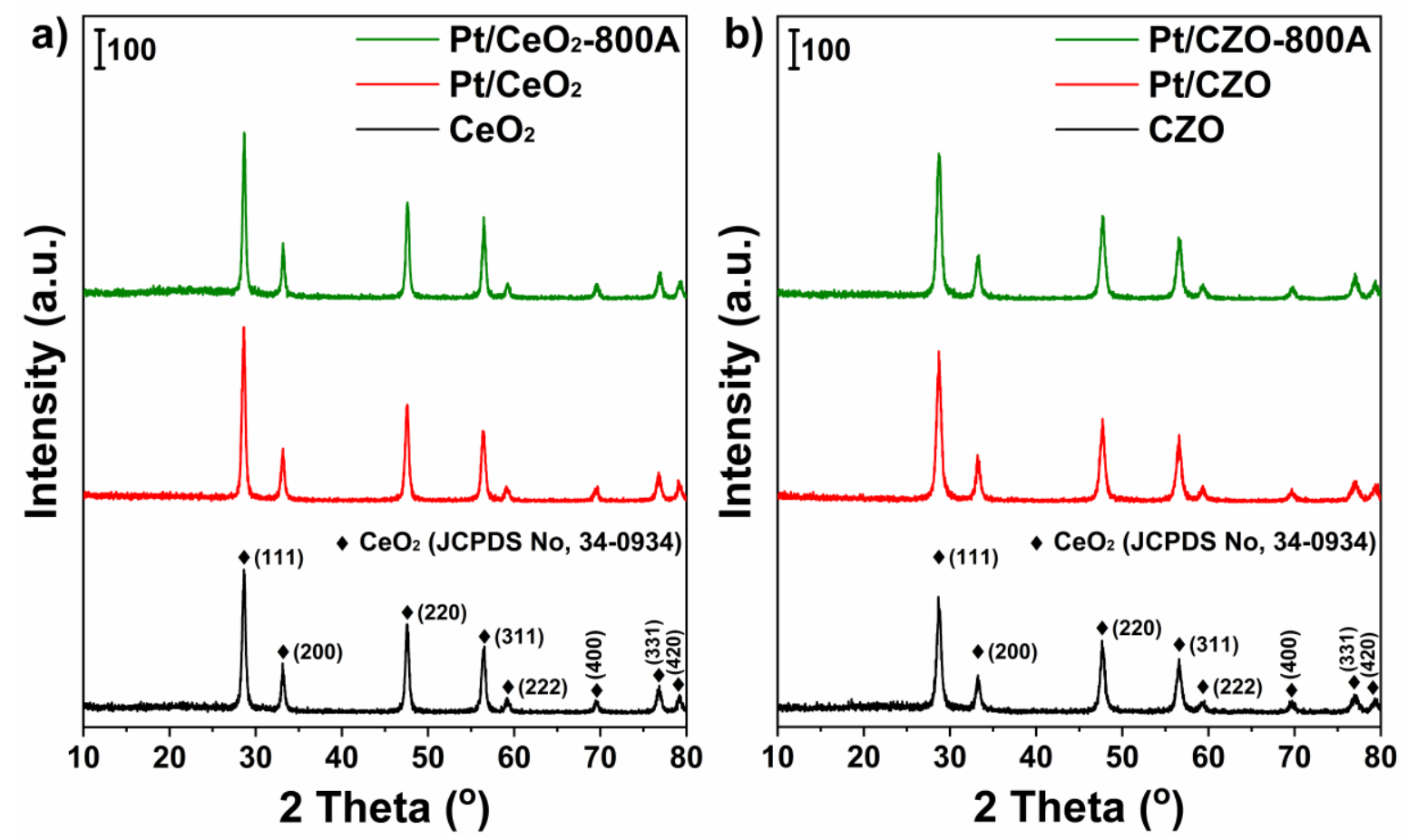

Figure S1. XRD patterns of a) $\mathrm{CeO}_{2}, \mathrm{Pt} / \mathrm{CeO}_{2}$ and $\mathrm{Pt} / \mathrm{CeO}_{2}-800 \mathrm{~A}$; b) $\mathrm{CZO}, \mathrm{Pt} / \mathrm{CZO}$ and Pt/CZO-800A. 
Supporting Information

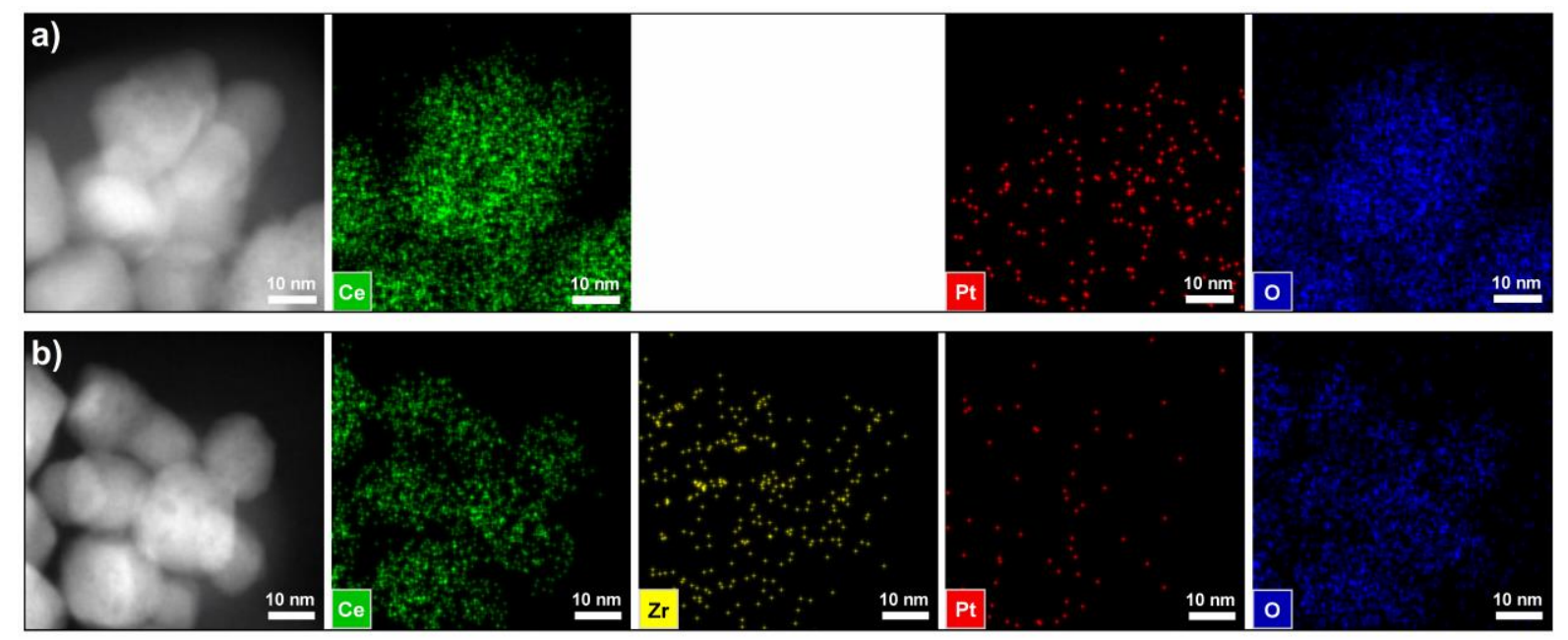

Figure S2. HAADF-STEM and EDS mapping images for a) $\mathrm{Pt} / \mathrm{CeO}_{2}-800 \mathrm{~A}$ and b) $\mathrm{Pt} / \mathrm{CZO}-$ 800A. 
Supporting Information
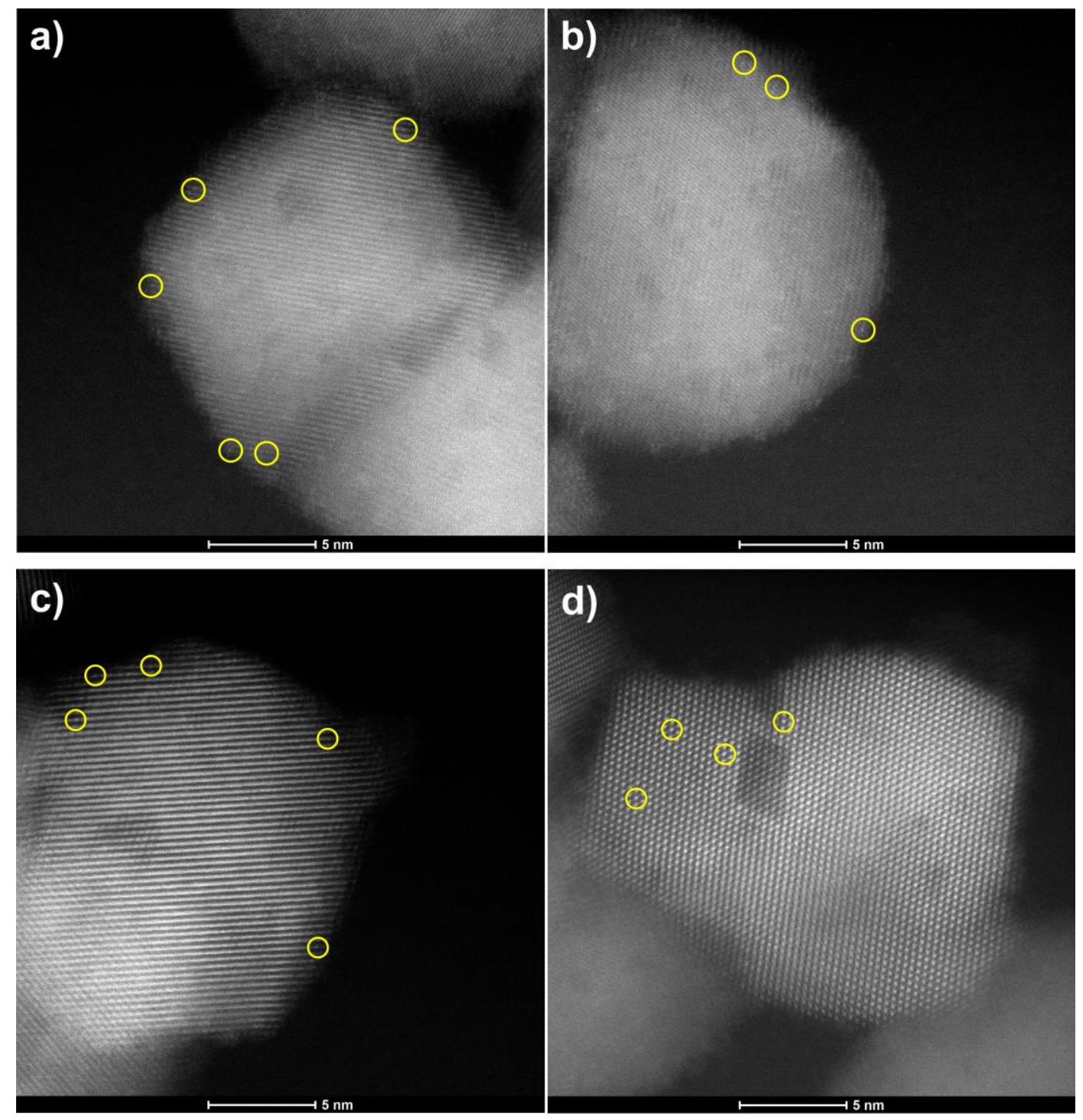

Figure S3. HAADF-STEM images of a, b) $\mathrm{Pt} / \mathrm{CeO}_{2}-800 \mathrm{~A}$ and $\mathbf{c}$, d) $\mathrm{Pt} / \mathrm{CZO}-800 \mathrm{~A}$. Note:

Some of Pt single sites were marked in yellow circles. 

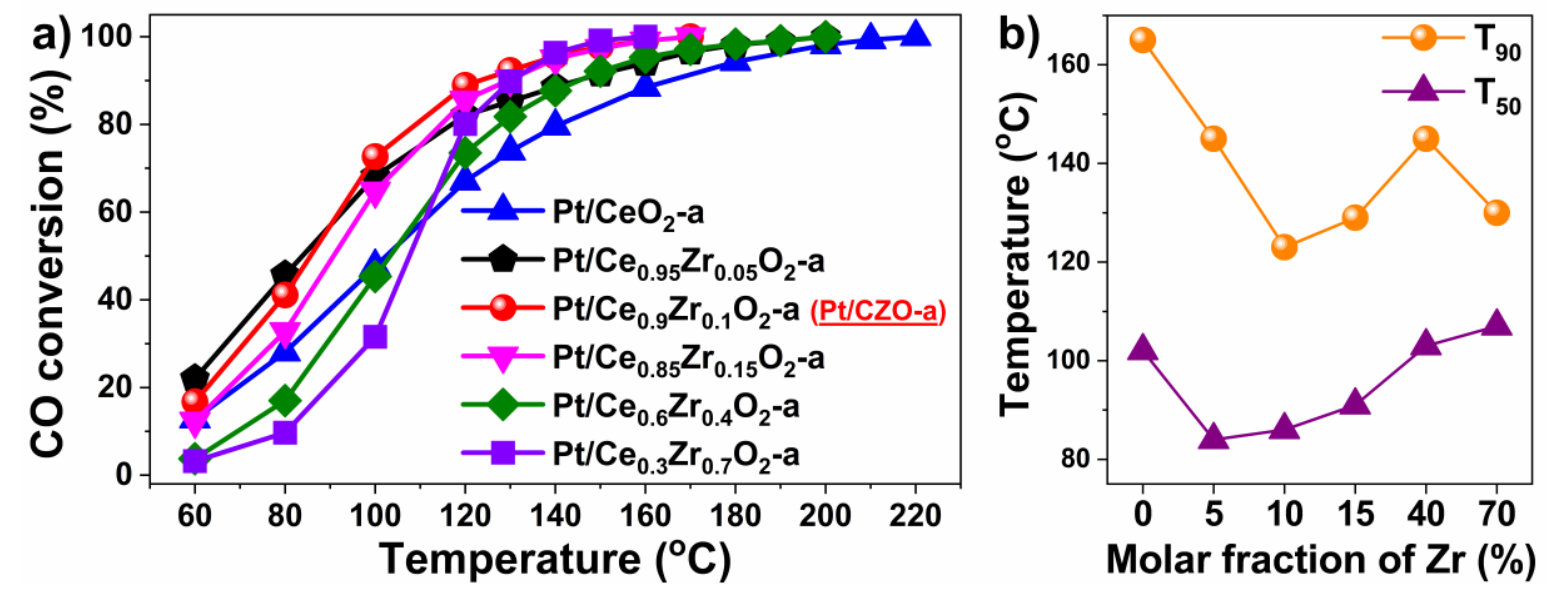

Figure S4. a) $\mathrm{CO}$ oxidation activity on $\mathrm{Pt} / \mathrm{Ce}_{x} \mathrm{Zr}_{1-x} \mathrm{O} 2-\mathrm{a}$; b) Temperatures at which the $\mathrm{CO}$ conversion reaches 50\% $\left(\mathrm{T}_{50}\right)$ and $90 \%\left(\mathrm{~T}_{90}\right)$ on $\mathrm{Pt} / \mathrm{Ce}_{x} \mathrm{Zr}_{1-x} \mathrm{O}_{2-\mathrm{a}} \cdot\left(\left[\mathrm{O}_{2}\right]=[\mathrm{CO}]=1 \%, \mathrm{Ar}\right.$ balance, $\left.\mathrm{WHSV}=200,000 \mathrm{~mL} \cdot \mathrm{g}_{\mathrm{cat}}{ }^{-1} \cdot \mathrm{h}^{-1}\right)$ 


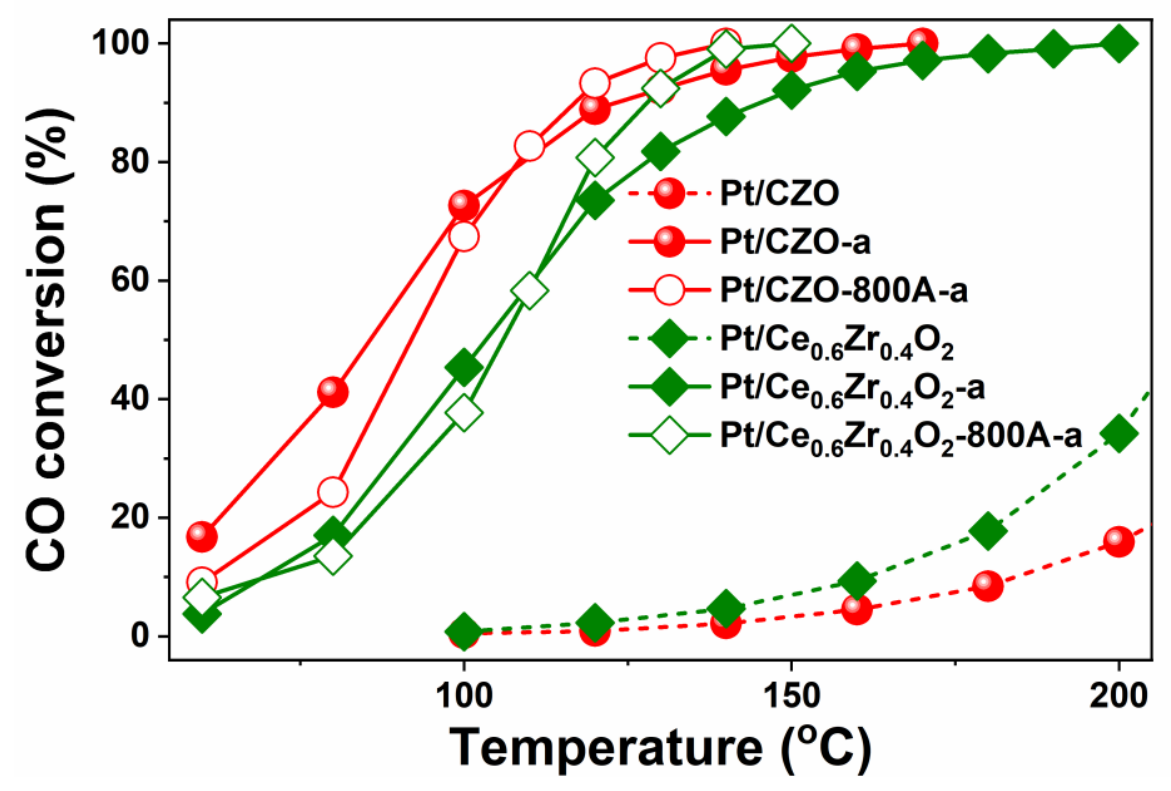

Figure S5. $\mathrm{CO}$ oxidation activity on $\mathrm{Pt} / \mathrm{CZO}\left(\mathrm{CZO}=\mathrm{Ce}_{0.9} \mathrm{Zr}_{0.1} \mathrm{O}_{2}\right), \mathrm{Pt} / \mathrm{CZO}-\mathrm{a}, \mathrm{Pt} / \mathrm{CZO}-800 \mathrm{~A}-$ a, $\mathrm{Pt} / \mathrm{Ce} 0.6 \mathrm{Zr}_{0.4} \mathrm{O}_{2}, \mathrm{Pt} / \mathrm{Ce} 0.6 \mathrm{Zr}_{0.4} \mathrm{O}_{2}-\mathrm{a}$ and $\mathrm{Pt} / \mathrm{Ce}_{0.6} \mathrm{Zr}_{0.4} \mathrm{O}_{2}-800 \mathrm{~A}-\mathrm{a} .\left(\left[\mathrm{O}_{2}\right]=[\mathrm{CO}]=1 \%, \mathrm{Ar}\right.$ balance, $\left.\mathrm{WHSV}=200,000 \mathrm{~mL} \cdot \mathrm{g}_{\mathrm{cat}}{ }^{-1} \cdot \mathrm{h}^{-1}\right)$ 


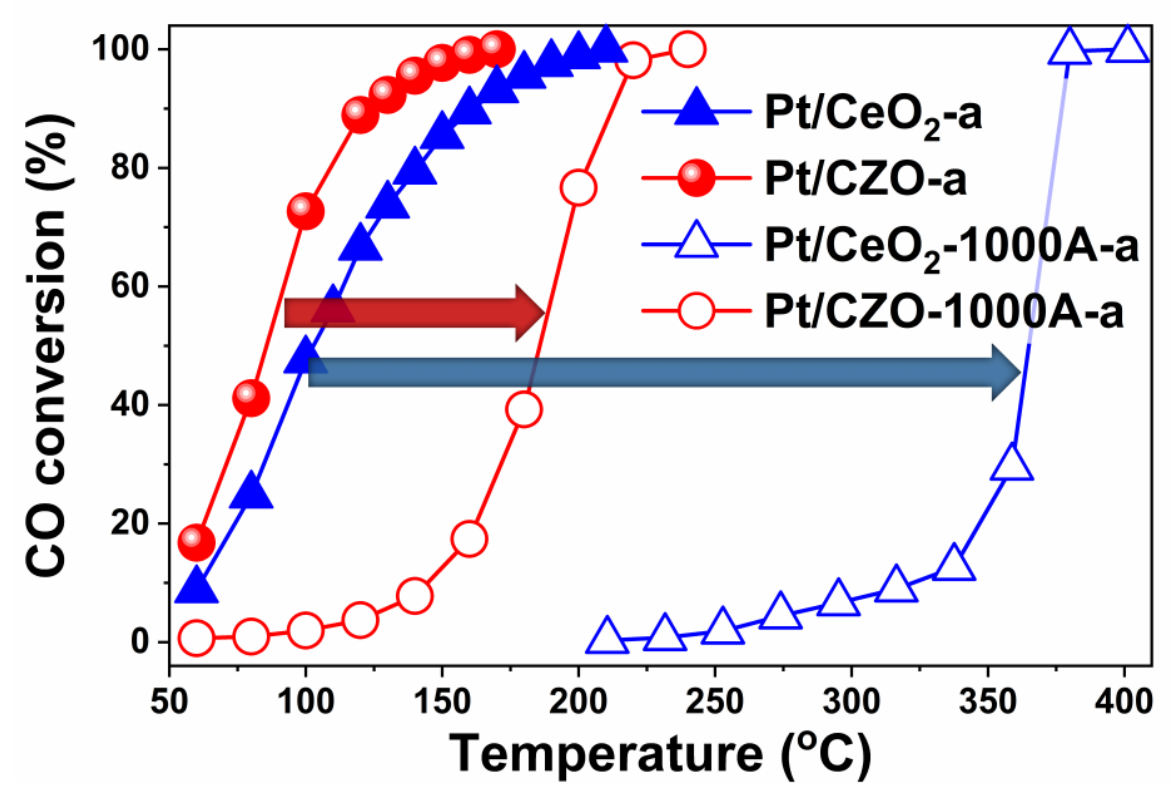

Figure S6. $\mathrm{CO}$ oxidation activity on $\mathrm{Pt} / \mathrm{CeO}_{2}-\mathrm{a}, \mathrm{Pt} / \mathrm{CZO}-\mathrm{a}, \mathrm{Pt} / \mathrm{CeO}_{2}-1000 \mathrm{~A}-\mathrm{a}$ and $\mathrm{Pt} / \mathrm{CZO}-$ $1000 \mathrm{~A}-\mathrm{a} .\left([\mathrm{CO}]=\left[\mathrm{O}_{2}\right]=1 \%, \mathrm{WHSV}=200,000 \mathrm{~mL} \cdot \mathrm{g}_{\text {cat. }}{ }^{-1} \cdot \mathrm{h}^{-1}\right)$ 


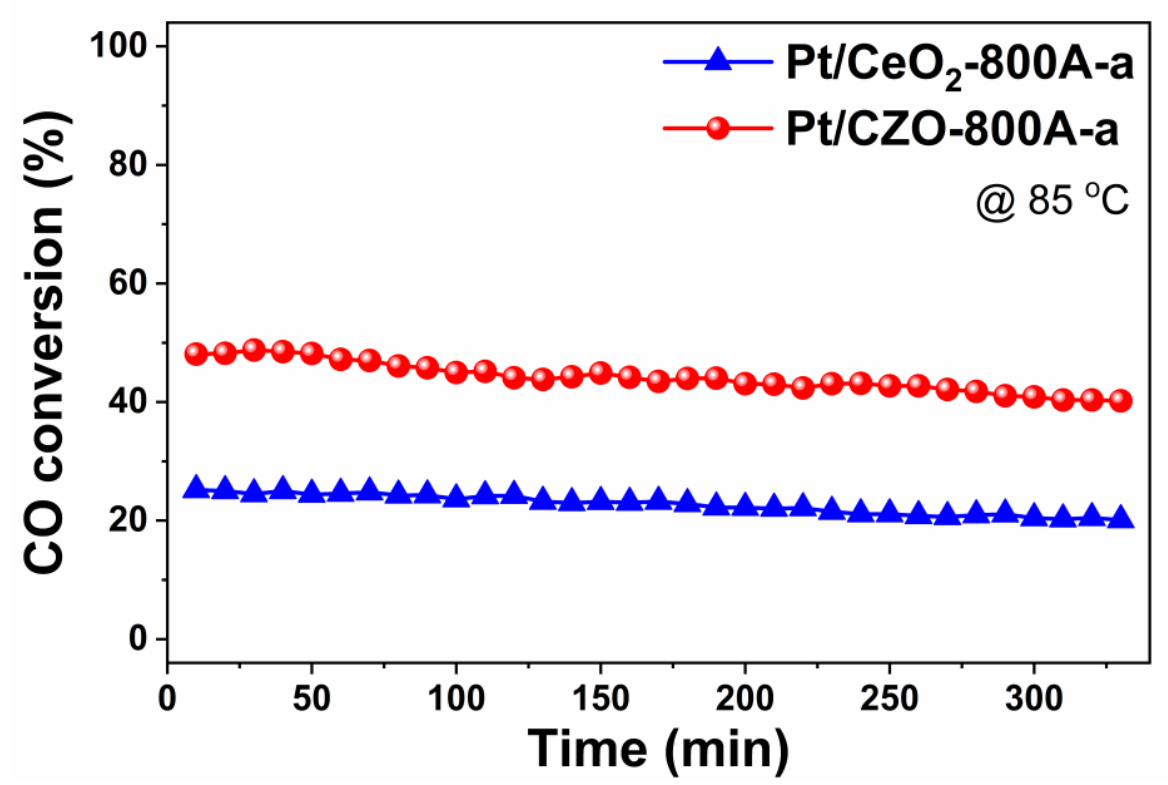

Figure S7. CO oxidation activity on $\mathrm{Pt} / \mathrm{CeO}_{2}-800 \mathrm{~A}-\mathrm{a}$ and $\mathrm{Pt} / \mathrm{CZO}-800 \mathrm{~A}-\mathrm{a}$ at $85^{\circ} \mathrm{C}$ as a function of time. $\left([\mathrm{CO}]=\left[\mathrm{O}_{2}\right]=1 \%\right.$, WHSV $\left.=100,000 \mathrm{~mL} \cdot \mathrm{g}_{\text {cat. }}{ }^{-1} \cdot \mathrm{h}^{-1}\right)$ 

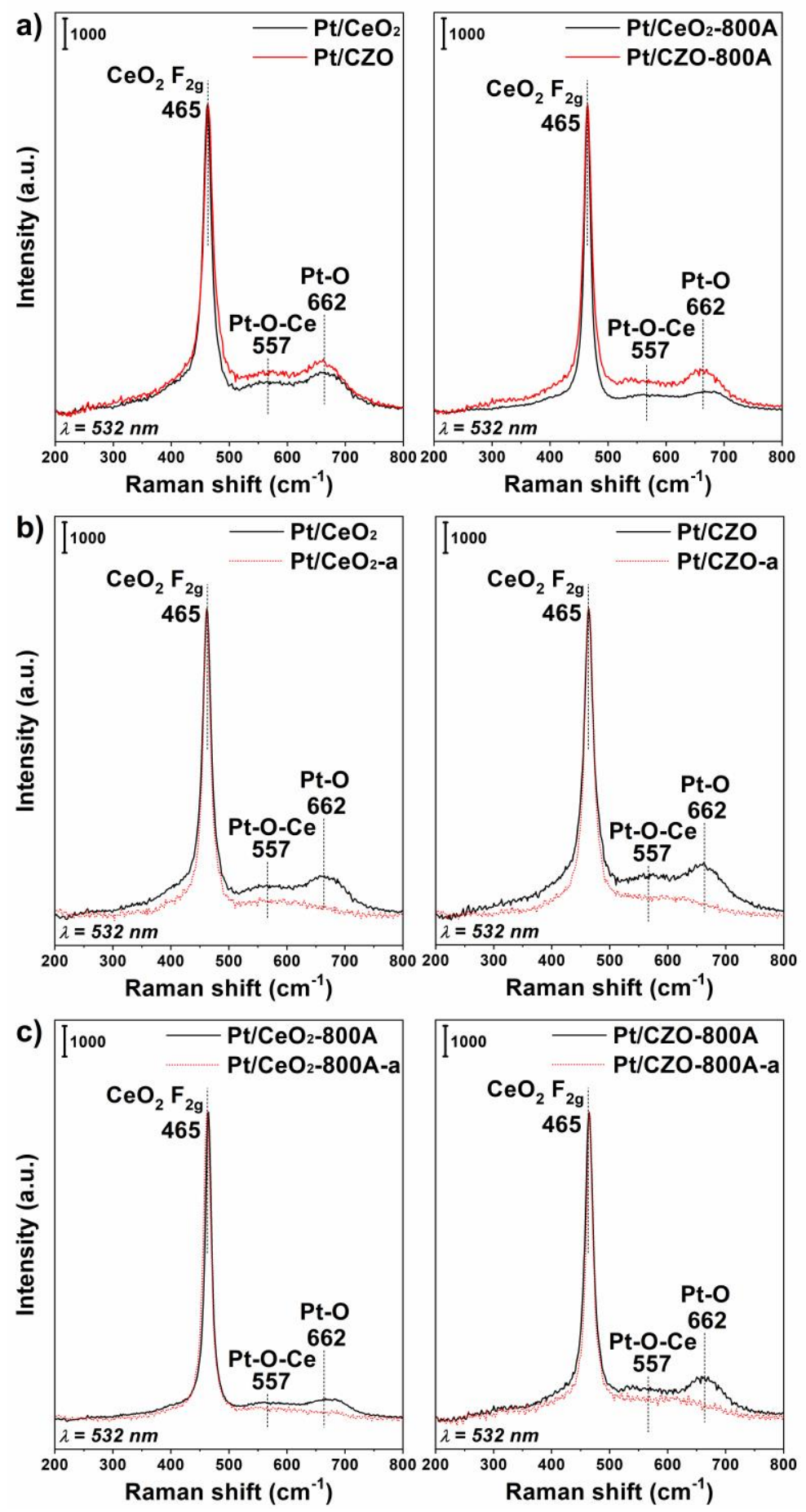

Figure S8. Raman spectra for a) fresh and aged catalysts: $\mathrm{Pt} / \mathrm{CeO}_{2}$ vs. $\mathrm{Pt} / \mathrm{CZO}$; b) fresh and

c) aged catalysts before and after activation treatment. 

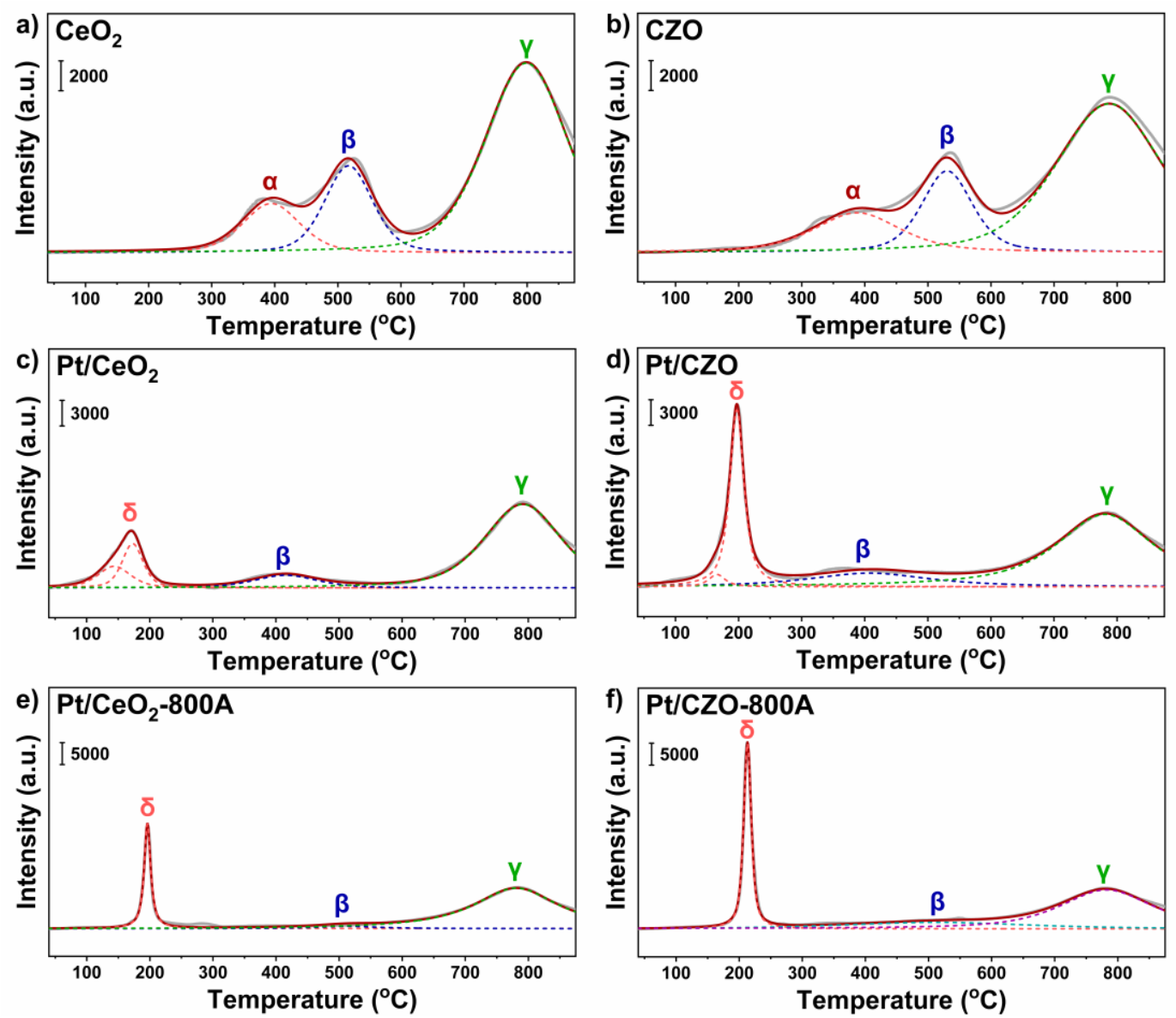

Figure S9. $\mathrm{H}_{2}-\mathrm{TPR}$ profiles of a) $\mathrm{CeO}_{2}$ and $\mathrm{CZO}$, b) fresh $\mathrm{Pt} / \mathrm{CeO}_{2}$ and $\mathrm{Pt} / \mathrm{CZO}$, c) aged $\mathrm{Pt} / \mathrm{CeO}_{2}-800 \mathrm{~A}$ and $\mathrm{Pt} / \mathrm{CZO}-800 \mathrm{~A}$. 


\section{Supporting Information}

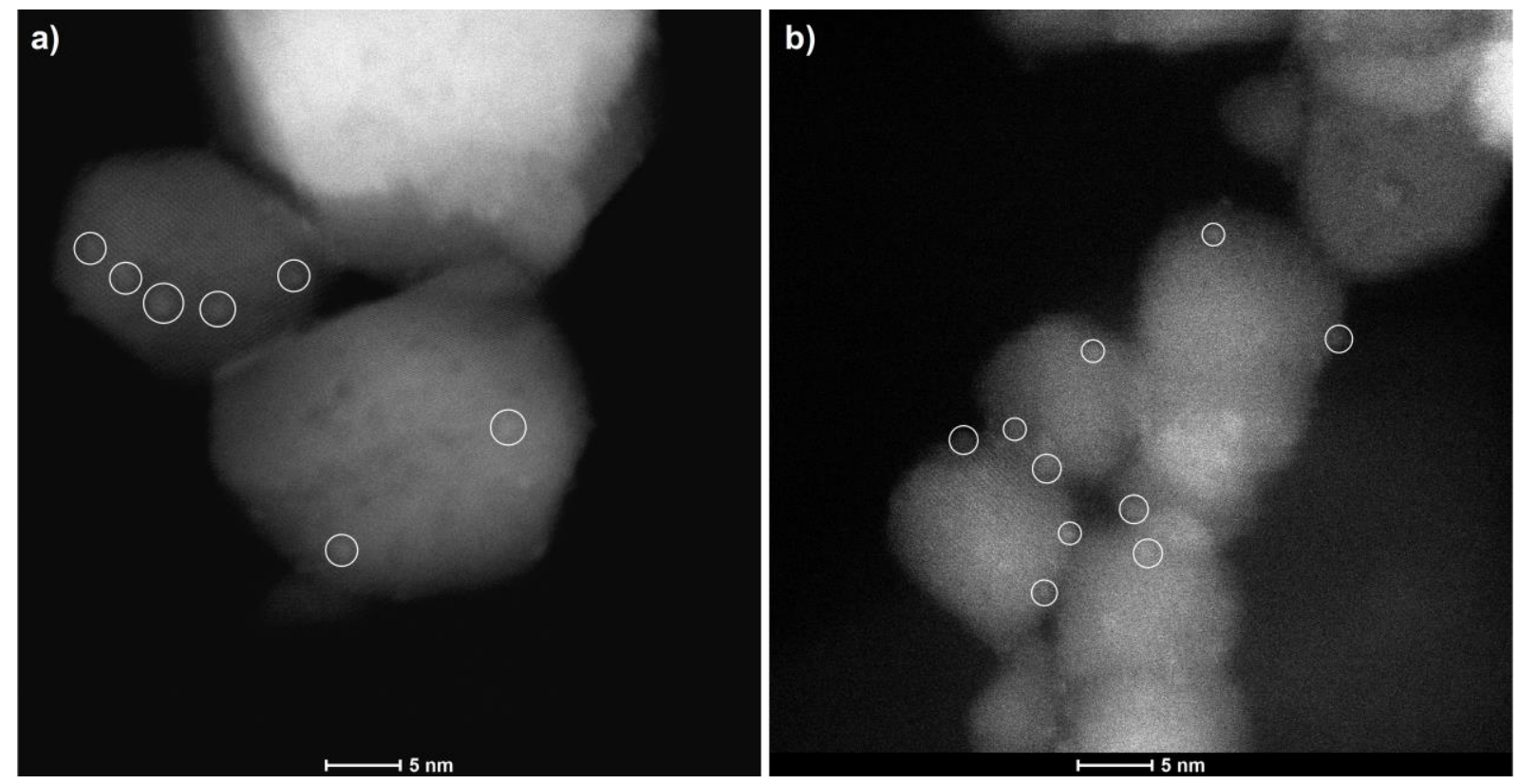

Figure S10. HAADF-STEM images of a) $\mathrm{Pt} / \mathrm{CeO}_{2}-800 \mathrm{~A}-\mathrm{a}$ and b) $\mathrm{Pt} / \mathrm{CZO}-800 \mathrm{~A}-\mathrm{a}$. Note:

Small Pt clusters are marked in white circles. 

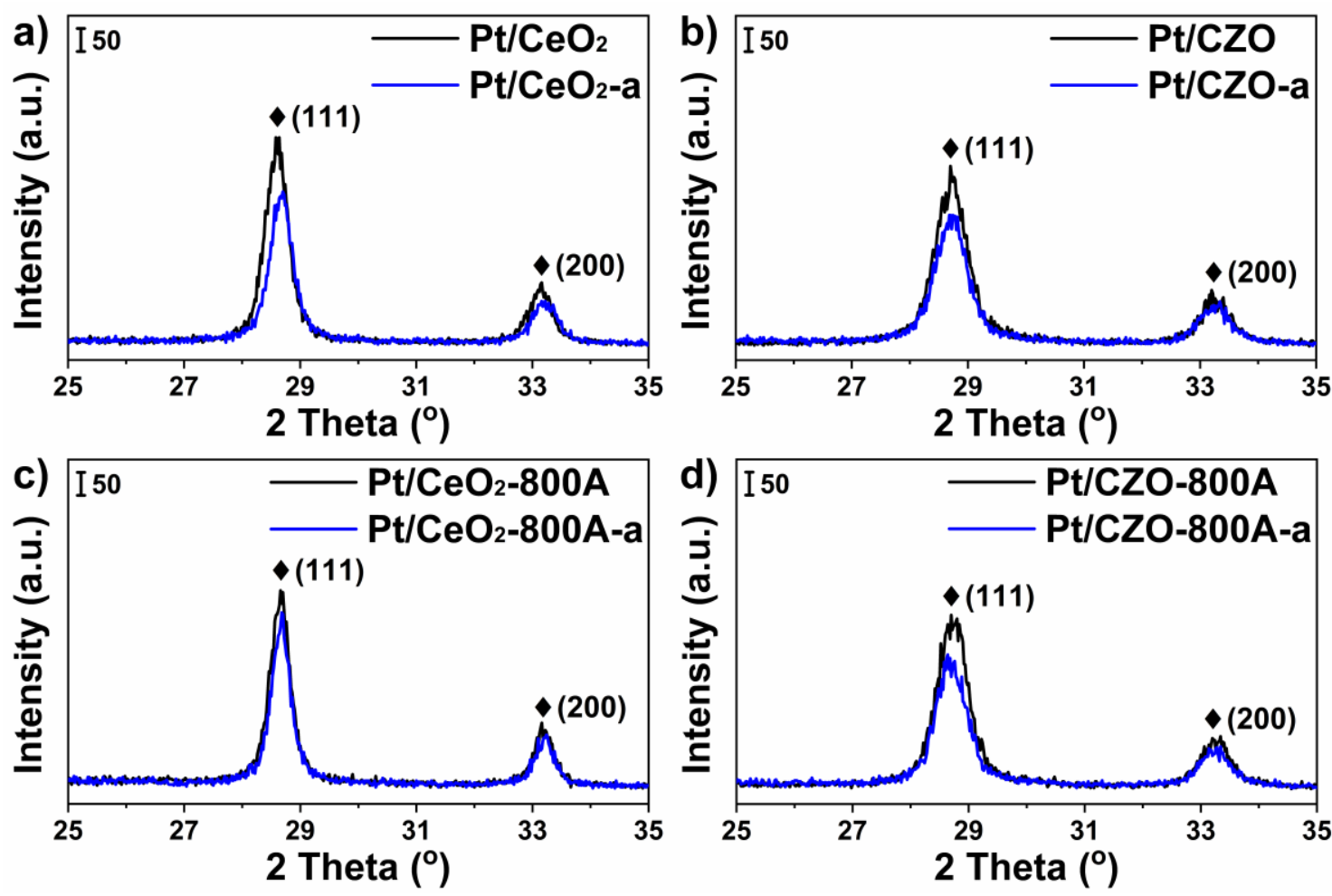

Figure S11 XRD patterns for a) $\mathrm{Pt} / \mathrm{CeO}_{2}$ and b) $\mathrm{Pt} / \mathrm{CZO}$, c) $\mathrm{Pt} / \mathrm{CeO}_{2}-800 \mathrm{~A}$ and d) $\mathrm{Pt} / \mathrm{CZO}$ $800 \mathrm{~A}$ before and after activation $\left(10 \% \mathrm{H}_{2}\right.$ at $400{ }^{\circ} \mathrm{C}$ for $\left.1 \mathrm{~h}\right)$. 


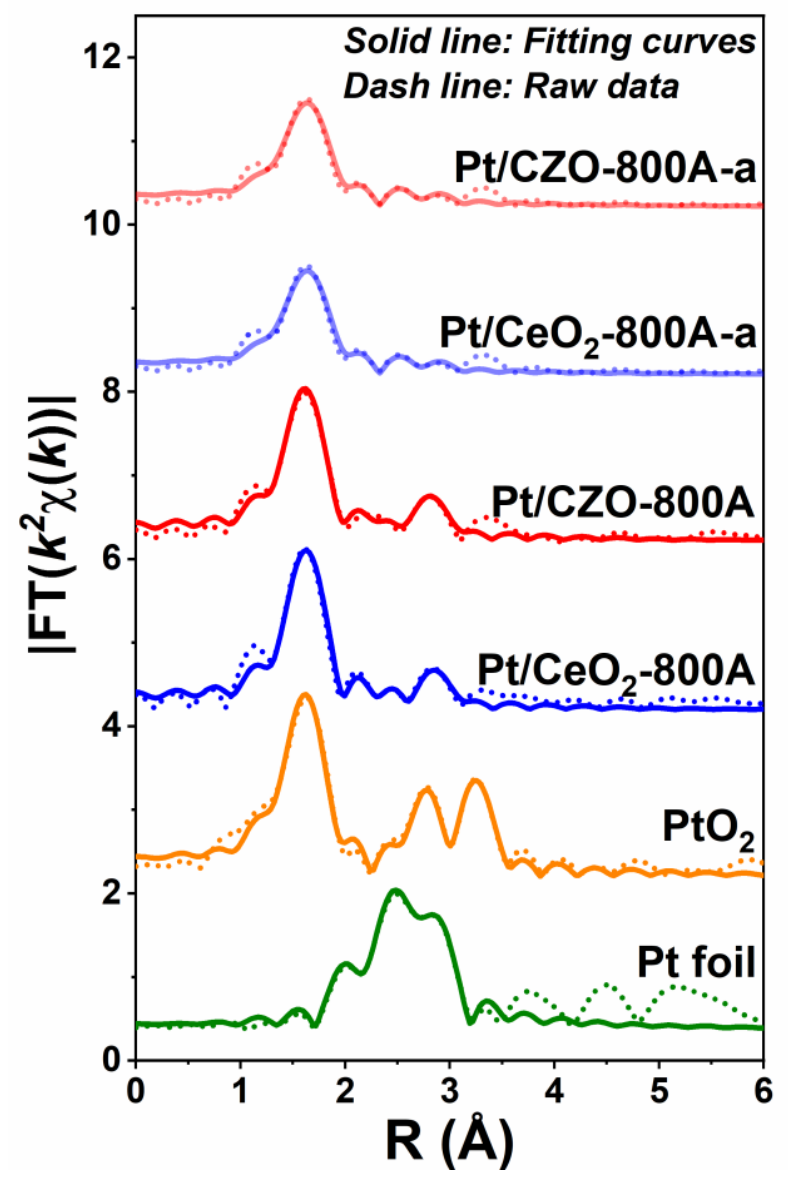

Figure S12. EXAFS fitting curves in R-space at the Pt-L3 edge. Dash lines represent the raw data and solid lines represent the fitting curves. 


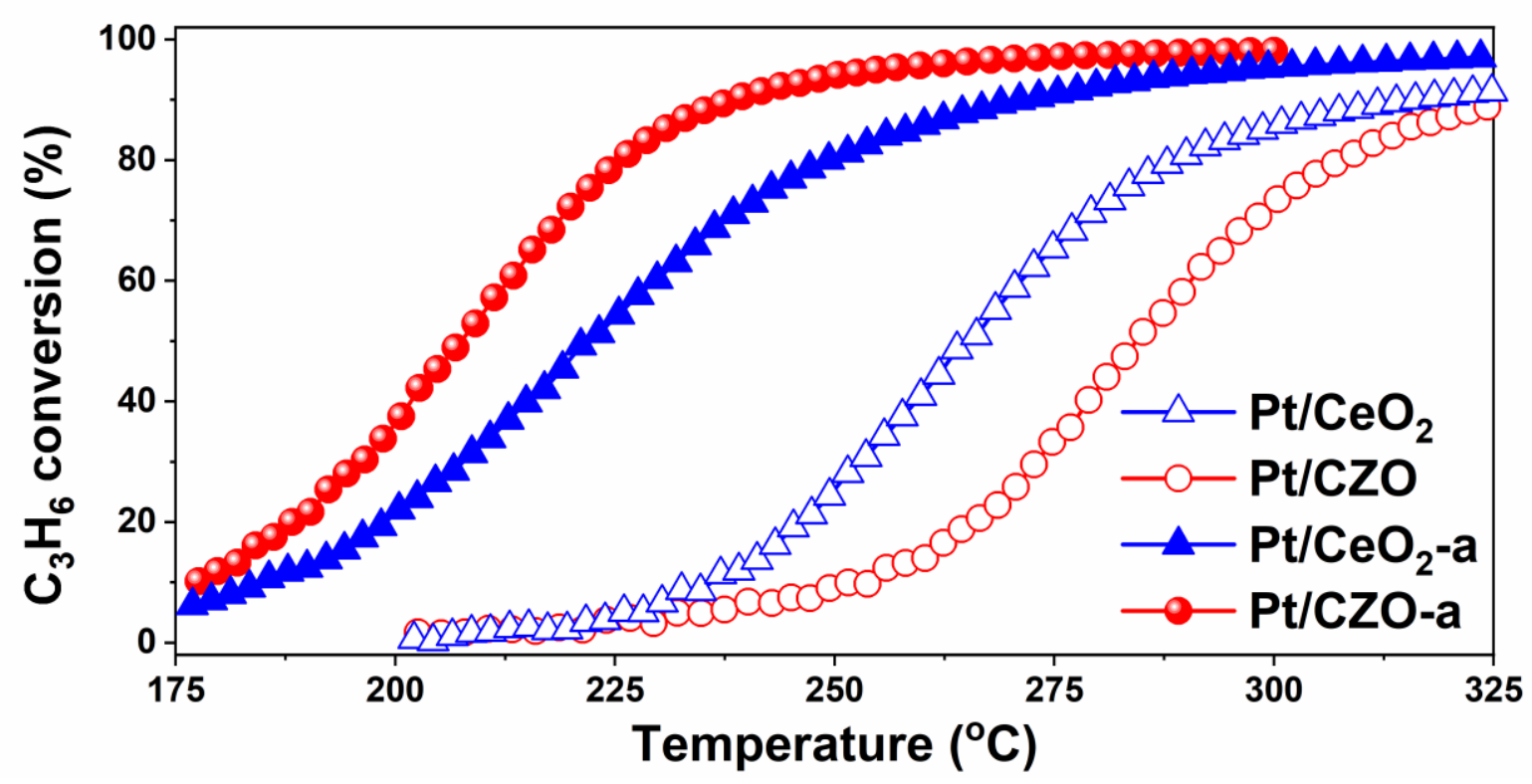

Figure S13. $\mathrm{C}_{3} \mathrm{H}_{6}$ oxidation activity on $\mathrm{Pt} / \mathrm{CeO}_{2}$ and $\mathrm{Pt} / \mathrm{CZO}$ were pretreated with air at 300 ${ }^{\circ} \mathrm{C}$ or activated with $10 \% \mathrm{H}_{2}$ at $500{ }^{\circ} \mathrm{C}$. 


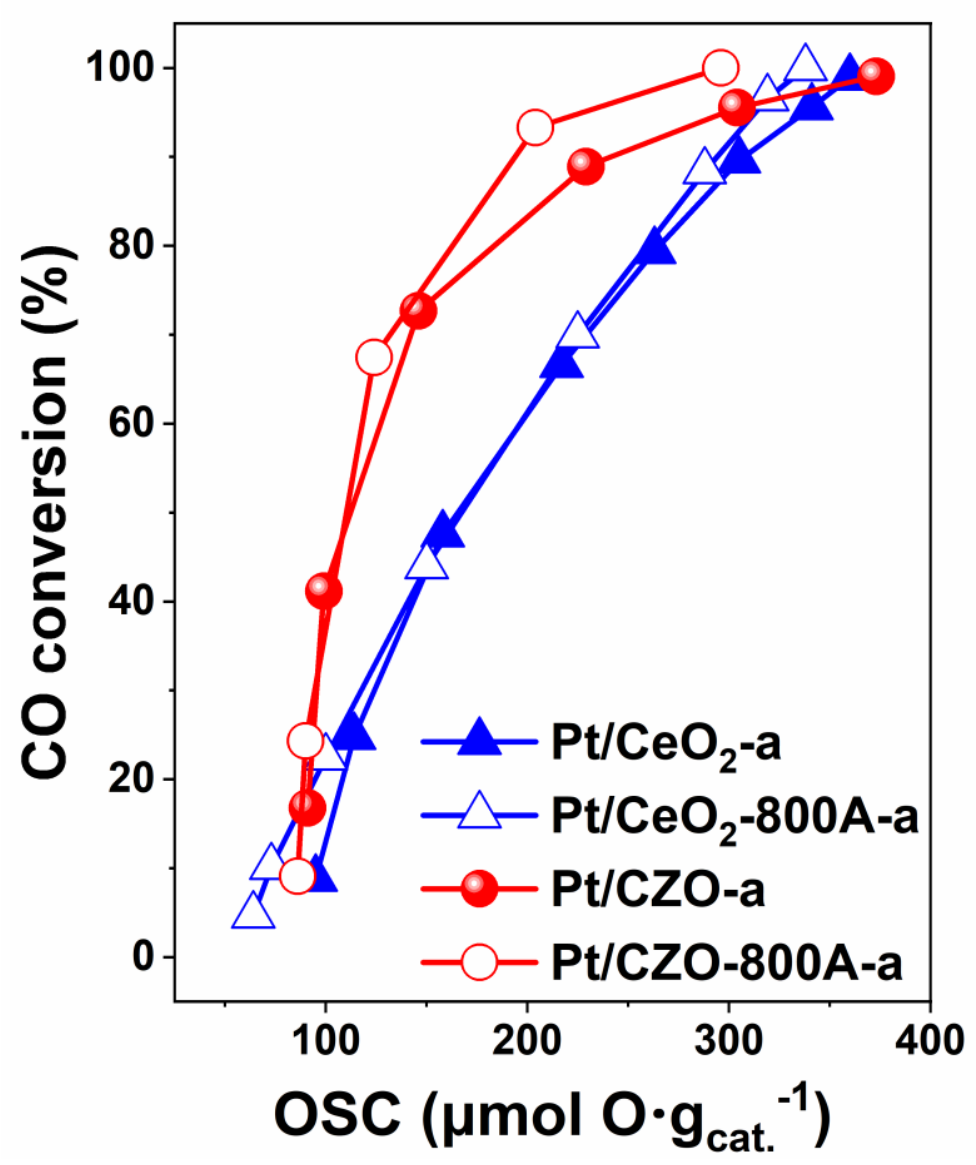

Figure S14. The relationship between $\mathrm{CO}$ conversion and $\mathrm{OSC}$ functions on $\mathrm{Pt} / \mathrm{CeO}_{2}-\mathrm{a}$, $\mathrm{Pt} / \mathrm{CeO}_{2}-800 \mathrm{~A}-\mathrm{a}, \mathrm{Pt} / \mathrm{CZO}-\mathrm{a}$ and Pt/CZO-800A-a. 


\section{Supporting Information}

\section{Supplementary References}

[1] W. Tan, J. Wang, S. Yu, A. Liu, L. Li, K. Guo, Y. Luo, S. Xie, F. Gao, F. Liu, L. Dong, Morphology-Sensitive Sulfation Effect on Ceria Catalysts for $\mathrm{NH}_{3}-\mathrm{SCR}$, Top. Catal., 2020, 63, 932-943. http://doi.org/10.1007/s11244-020-01342-8.

[2] L.R. Borges, A.G.M. da Silva, A.H. Braga, L.M. Rossi, M.A. Suller Garcia, P. Vidinha, Towards the Effect of $\mathrm{Pt}^{0} / \mathrm{Pt}^{\delta+}$ and $\mathrm{Ce}^{3+}$ Species at the Surface of $\mathrm{CeO}_{2}$ Crystals: Understanding the Nature of the Interactions under CO Oxidation Conditions, ChemCatChem, 2021, 13, 1340 1354. https://doi.org/10.1002/cctc.202001621.

[3] L. Nie, D. Mei, H. Xiong, B. Peng, Z. Ren, X.I.P. Hernandez, A. DeLaRiva, M. Wang, M.H. Engelhard, L. Kovarik, Activation of Surface Lattice Oxygen in Single-Atom Pt/CeO 2 for LowTemperature CO Oxidation, Science, 2017, 358, 1419-1423. http://doi.org/10.1126/science.aao2109.

[4] R. Mandapaka, G. Madras, Zinc and Platinum Co-Doped Ceria for WGS and CO Oxidation, Appl. Catal., B, 2017, 211, 137-147. https://doi.org/10.1016/j.apcatb.2017.04.044.

[5] X.I. Pereira-Hernández, A. DeLaRiva, V. Muravev, D. Kunwar, H. Xiong, B. Sudduth, M. Engelhard, L. Kovarik, E.J.M. Hensen, Y. Wang, A.K. Datye, Tuning Pt-CeO2 Interactions by High-Temperature Vapor-Phase Synthesis for Improved Reducibility of Lattice Oxygen, Nat. Commun. 2019, 10, 1358. http://doi.org/10.1038/s41467-019-09308-5.

[6] L. Fan, K. Wang, K. Xu, Z. Liang, H. Wang, S.-F. Zhou, G. Zhan, Structural Isomerism of Two Ce-BTC for Fabricating $\mathrm{Pt} / \mathrm{CeO}_{2}$ Nanorods toward Low-Temperature CO Oxidation, Small, 2020, 16, 2003597. https://doi.org/10.1002/smll.202003597.

[7] Z. Guo, Q. You, L. Song, G. Sun, G. Chen, C. Li, X. Yang, X. Hu, X. Jiang, Highly Dispersed Pt Species Anchored onto $\mathrm{NH}_{2}$-Ce-MOFs and their Derived Mesoporous Catalysts for CO Oxidation, Nanoscale, 2021, 13, 117-123. https://doi.org/10.1039/D0NR05626J.

[8] S. Guo, Y. Zhao, C. Wang, H. Jiang, G.J. Cheng, A Single-Atomic Noble Metal Enclosed Defective MOF via Cryogenic UV Photoreduction for CO Oxidation with Ultrahigh Efficiency and Stability, ACS Appl. Mater. Interfaces, 2020, 12, 26068-26075. https://doi.org/10.1021/acsami.0c06898.

[9] M. Yoo, Y.-S. Yu, H. Ha, S. Lee, J.-S. Choi, S. Oh, E. Kang, H. Choi, H. An, K.-S. Lee, J.Y. Park, R. Celestre, M.A. Marcus, K. Nowrouzi, D. Taube, D.A. Shapiro, W. Jung, C. Kim, H.Y. Kim, A Tailored Oxide Interface Creates Dense Pt Single-Atom Catalysts with High Catalytic Activity, Energy Environ. Sci. 2020, 13 (4), 1231-1239. https://doi.org/10.1039/C9EE03492G.

[10] X. Yang, X. Cheng, J. Ma, Y. Zou, W. Luo, Y. Deng, Large-Pore Mesoporous $\mathrm{CeO}_{2}-\mathrm{ZrO}_{2}$ Solid Solutions with In-Pore Confined Pt Nanoparticles for Enhanced CO Oxidation, Small, 2019, 15, 1903058. https://doi.org/10.1002/smll.201903058. 